1920.] ROOTS AND POLES OF RATIONAL FUNCTIONS.

are satisfied, the inequalities

$$
\left|g_{n}(x)-g_{n}\left(\xi_{i}\right)\right| \leqq \max |f| \cdot V_{y}\left[K(x, y)-K\left(\xi_{i}, y\right)\right]
$$

and inequality (7) will imply that

$$
(n=1,2, \cdots) \text {, }
$$

$$
\begin{aligned}
\left|g_{n}(x)-g_{n+m}(x)\right| \leqq 2 & {[\max |f|+1] \epsilon } \\
& \left(n \geqq N^{\prime} ; m=1,2, \cdots\right) .
\end{aligned}
$$

Consequently these $g$ 's must be uniformly convergent, and the transformation must be completely continuous.

If condition $B$ is not satisfied there must be an $\epsilon>0$ and an infinite sequence of $x$ 's such that

$$
V_{y}\left[K\left(x_{m}, y\right)-K\left(x_{n}, y\right)\right] \geqq \epsilon \quad(m \neq n) .
$$

It follows from the principal theorem of the first section, that if $V_{y} K$ is bounded uniformly, a subset of these $x$ 's must make $K\left(x_{n}, y\right)$ convergent. As no subset of them can satisfy equation (4), condition $A$ cannot be satisfied. Therefore when $A$ is satisfied $B$ must be, and since $A$ is necessary and $B$ sufficient, $B$ must be necessary and $A$ sufficient also.

Trinity College, HartFord, Conn.

\title{
ON THE RELATION OF THE ROOTS AND POLES OF A RATIONAL FUNCTION TO THE ROOTS OF ITS DERIVATIVE.
}

BY MR. BEN-ZION LINFIELD.

(Read before the American Mathematical Society December 30, 1919.)

1. F. Lucas, Journal de l'Ecole polytechnique, 1879, gave a mechanical proof of the following theorem:

The roots of the derivative of a cubic are the foci of the maximum ellipse inscribed in the triangle whose vertices are the roots of the cubic.

Professor Maxime Bôcher gave a simple proof of this theorem, Annals of Mathematics, volume 7, page 70, 1892. Here he made observations on the general theorem concerning the polynomial of $n$th degree and asked the question, "Could 
not the first of these propositions be brought into connection with the focal properties of the higher plane curves?" Other demonstrations of Lucas's theorem have been given, but the writer is not aware of Professor Bôcher's question having been answered. It is the object of this note to answer this question by the following:

2. THEOREM. The roots of the derivative of the rational function

$$
f(z) \equiv \prod_{i=1}^{n}\left(z-z_{i}\right)^{\mu_{i}}
$$

are the multiple roots of $f(z)$ to one lower order and the foci of $a$ curve of class $n-1$ which touches each segment $z_{i} z_{j}(i \neq j$, $=1,2, \cdots, n)$ at a point dividing it in the ratio of $\mu_{i}$ to $\mu_{j}$.

3. Let

$$
\alpha_{i} \equiv u x_{i}+v y_{i}+w \quad(i=1,2, \cdots, n)
$$

be the line equation of the point $P_{i} \equiv\left(x_{i}, y_{i}\right)$, where $z_{i} \equiv x_{i}$ $+y_{i} \sqrt{-1}$ are the roots and poles of the rational function $f(z)$. Then,

$$
\Pi \equiv \alpha_{1} \alpha_{2} \cdots \alpha_{n}=0
$$

is the line equation of the $n$ points $x_{i}, y_{i}$.

$$
\varphi(u, v, w) \equiv \sum_{i=1}^{n} \mu_{i} \frac{\partial \Pi}{\partial \alpha_{i}}=0
$$

is the tangential (line) equation of a curve of class $n-1$ tangent to the segments $P_{i} P_{j}$.

The point of contact of an arbitrary tangent to (2) whose coordinates are $u, v, w$, is given by equations

Also from (2)

$$
\frac{X}{\frac{\partial \varphi}{\partial u}}=\frac{Y}{\frac{\partial \varphi}{\partial v}}=\frac{1}{\frac{\partial \varphi}{\partial w}} .
$$

$$
\begin{aligned}
& \frac{\partial \varphi}{\partial u}=\Sigma\left(\mu_{j} x_{i}+\mu_{i} x_{j}\right) \frac{\partial^{2} \Pi}{\partial \alpha_{i} \partial \alpha_{j}}, \\
& \frac{\partial \varphi}{\partial v}=\Sigma\left(\mu_{j} y_{i}+\mu_{i} y_{j}\right) \frac{\partial^{2} \Pi}{\partial \alpha_{i} \partial \alpha_{j}}, \\
& \frac{\partial \varphi}{\partial w}=\Sigma\left(\mu_{i}+\mu_{j}\right) \frac{\partial^{2} \Pi}{\partial \alpha_{i} \partial \alpha_{j}} .
\end{aligned}
$$


Therefore the coordinates of the point of contact of the tangent $(u, v, w)$ with (2) are

$$
\begin{gathered}
X=\frac{\Sigma\left(\mu_{j} x_{i}+\mu_{i} x_{j}\right) \partial^{2} \Pi / \partial \alpha_{i} \partial \alpha_{j}}{\Sigma\left(\mu_{i}+\mu_{j}\right) \partial^{2} \Pi / \partial \alpha_{i} \partial \alpha_{j}}, \\
Y=\frac{\Sigma\left(\mu_{j} y_{i}+\mu_{i} y_{j}\right) \partial^{2} \Pi / \partial \alpha_{i} \partial \alpha_{j}}{\Sigma\left(\mu_{i}+\mu_{j}\right) \partial^{2} \Pi / \partial \alpha_{i} \partial \alpha_{j}} .
\end{gathered}
$$

Let the tangent $(u, v, w)$ coincide with the tangent through the two points $P_{p}$ and $P_{q}$. Then

$$
\frac{\partial^{2} \Pi}{\partial \alpha_{i} \partial \alpha_{j}}=0 \quad(\text { when } i \neq p, j \neq q),
$$

since $\alpha_{p}=0, \alpha_{q}=0$ and $\alpha_{p}$ or $\alpha_{q}$ occurs in each of these second derivatives except $\partial^{2} \Pi / \partial \alpha_{p} \partial \alpha_{q}$. Hence the coordinates of the point of contact of (2) with $P_{p} P_{q}$ are

$$
X=\frac{\mu_{q} x_{p}+\mu_{p} x_{q}}{\mu_{q}+\mu_{p}}, \quad Y=\frac{\mu_{q} y_{p}+\mu_{p} y_{q}}{\mu_{q}+\mu_{p}},
$$

dividing the segment $P_{p} P_{q}$ in the ratio $\mu_{p}: \mu_{q}$.

4. The foci of the curve $\varphi(u, v, w)=0$ are the roots of the polynomial $\varphi(-1,-\sqrt{-1}, z)^{*}$ where $z \equiv x+y \sqrt{-1}$.

Thus in (2)

Also

$$
\alpha_{i} \equiv z-z_{i}, \quad \Pi \equiv \prod_{i=1}^{n}\left(z-z_{i}\right) .
$$

$$
\varphi(-1,-\sqrt{-1}, z) \equiv \sum_{j=1}^{n} \mu_{j} \frac{\prod_{i=1}^{n}\left(z-z_{i}\right)}{z-z_{i}} .
$$

But the derivative of $f(z)$ can be written

$$
f^{\prime}(z)=\prod_{i=1}^{n}\left(z-z_{i}\right)^{\mu_{i}-1} \cdot \sum \mu_{j} \frac{\prod_{i=1}^{n}\left(z-z_{i}\right)}{z-z_{j}},
$$

which establishes the theorem, $\S 2$, as enunciated.

5 . The present note is an abstract presenting the central theorem of a paper wherein the general curve $\varphi=0$ of class

* Salmon, Higher Plane Curves, 3d ed., § 141; also Emch, this BuLleTIN, vol. 25, pp. 157-161. 
$n-1$ is discussed and methods evolved for drawing it. We go no further here than to notice the interesting case when $n=3$, of which the theorem of Lucas is a particular case.

6. When $n=3$ the roots of the derivative of

$$
f(z) \equiv \prod_{i=1}^{3}\left(z-z_{i}\right)^{\mu_{i}}
$$

are the roots of $\prod_{1}^{3}\left(z-z_{i}\right)^{\mu_{i}-1}$ and the foci of

$$
\varphi \equiv \mu_{1} \alpha_{2} \alpha_{3}+\mu_{2} \alpha_{1} \alpha_{3}+\mu_{3} \alpha_{1} \alpha_{2}=0 .
$$

The curve is clearly of the second class and therefore a conic. The center $z_{c}$ is given by

or

$$
\frac{\partial}{\partial z} \Sigma \mu_{j} \frac{\Pi\left(z-z_{i}\right)}{z-z_{j}}=0,
$$

$$
z_{c}=\frac{\left(\mu_{2}+\mu_{3}\right) z_{1}+\left(\mu_{3}+\mu_{1}\right) z_{2}+\left(\mu_{1}+\mu_{2}\right) z_{3}}{2\left(\mu_{1}+\mu_{2}+\mu_{3}\right)} .
$$

When the powers $(\mu)$ are positive the conic $\varphi=0$ touches the sides of the triangle $z_{1}, z_{2}, z_{3}$ internally, and is therefore an ellipse inscribed in the triangle; the point of contact divides each side in the ratio of the powers of the vertices. If $\mu_{1}=\mu_{2}=\mu_{3}=1, f(z)$ becomes a cubic and the conic touches the sides of the triangle at their midpoints, which is Lucas's case.

When each power is not positive, it is only necessary to consider one as being negative, for changing the signs of all the powers does not effect $\varphi=0$.

If $\mu_{1}+\mu_{2}+\mu_{3}=0$, the conic is a parabola, the center being at infinity. It follows from a well-known theorem, the focus of a parabola touching the three sides of a triangle lies on the circumcircle of the triangle, that in this case the roots of the derivative of $f(z)$ lie on a circle through the roots of $f(z)$.

A conic is clearly an ellipse or hyperbola according as it passes through a point between or not between two parallel tangents, respectively.

The tangents parallel to the sides of the triangle are easily found, since the center is known and the point of touch on a 
side. Let the side $z_{1} z_{2}$ be the $x$-axis. The equation of the tangent parallel to this axis is

$$
y=\frac{\mu_{1}+\mu_{2}}{\mu_{1}+\mu_{2}+\mu_{3}} y_{3},
$$

with similar equations of the tangents parallel to the other two sides. Let $\mu_{3}$ be negative. The points of contact with the sides $z_{1} z_{3}$ and $z_{2} z_{3}$ lie between the $x$-axis and the parallel tangent or outside them according as

$$
\mu_{1}+\mu_{2}+\mu_{3} \lessgtr 0 \text {, }
$$

and the conic is accordingly an ellipse or hyperbola.

Hence if all the powers be positive $\varphi=0$ is an ellipse. If one of the powers be negative it is an ellipse, parabola or hyperbola according as their sum is less than, equal to or greater than zero. If two of the powers be negative it is an ellipse, parabola or hyperbola according as their sum is greater than, equal to or less than zero. In particular if $\mu_{1}+\mu_{3}=0$ the side $z_{1} z_{3}$ is an asymptote. If $\mu_{1}=\mu_{2}=-\mu_{3}$ the sides $z_{1} z_{3}$ and $z_{2} z_{3}$ are asymptotes. If in addition the triangle $z_{1} z_{2} z_{3}$ is isosceles having its vertex at $z_{3}$, the circle having $z_{3}$ for its center and passing through $z_{1}$ and $z_{2}$ passes through the foci of $\varphi=0$, for the altitude and half base of this triangle are the major and minor axes respectively.

UNIVERSITY OF VIRGINIA, May 1, 1919.

\section{MORITZ CANTOR, THE HISTORIAN OF MATHEMATICS.}

BY PROFESSOR FLORIAN CAJORI.

(Read before the San Francisco Section of the American Mathematical Society June 17, 1920.)

Professon Moritz Cantor died at Heidelberg on April 10, 1920 , in his ninety-first year, three days after the death of his only son. Considering his own long career and that of many other mathematicians, he could well remark on his eightieth birthday, "Die Mathematik gehört nicht gerade zu den ungesunden Handwerken."* His literary activity ex-

* Felix Müller, Der Mathematische Sternenhimmel des Jahres 1811, Leipzig und Berlin, 1911, p. 7. 